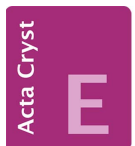

CRYSTALLOGRAPHIC COMMUNICATIONS

ISSN 2056-9890

Received 8 May 2015

Accepted 29 June 2015

Edited by G. Smith, Queensland University of Technology, Australia

Keywords: crystal structure; calcium benzoate; coordination polymer; $\mathrm{C}-\mathrm{H} \cdots \pi$ interactions

CCDC reference: 1409468

Supporting information: this article has

supporting information at journals.iucr.org/e

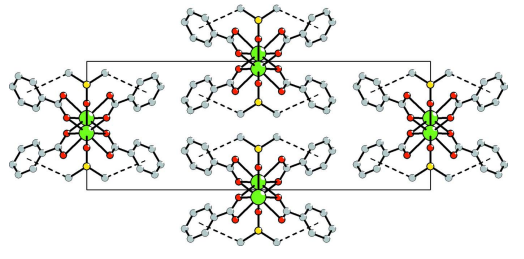

OPEN $\odot$ ACCESS

\section{Crystal structure of catena-poly[calcium-di- $\mu_{3^{-}}$ benzoato- $\kappa^{6} O, O^{\prime}: O-\mu_{2}$-(dimethyl sulfoxide)- $\left.\kappa^{2} \mathrm{O}: O\right]$}

\author{
Anna S. Voronova, ${ }^{a}$ Svitlana R. Petrusenko ${ }^{a *}$ and Evgeny Goreshnik ${ }^{b}$ \\ a Department of Inorganic Chemistry, Taras Shevchenko National University of Kyiv, Volodymyrska str. 64/13, 01601 \\ Kyiv, Ukraine, and ${ }^{\mathbf{b}}$ Department of Inorganic Chemistry and Technology, Jožef Stefan Institute, Jamova 39, 1000 \\ Ljubljana, Slovenia. *Correspondence e-mail: spetrusenko@yahoo.com
}

In the title complex, $\left[\mathrm{Ca}\left(\mathrm{C}_{7} \mathrm{H}_{5} \mathrm{O}_{2}\right)_{2}\left(\mathrm{C}_{2} \mathrm{H}_{6} \mathrm{OS}\right)\right]_{n}$, the $\mathrm{Ca}^{2+}$ ion (site symmetry m..) is surrounded by eight $\mathrm{O}$ atoms, six from two bridging-chelating tridentate benzoate carboxyl groups and two from a bridging dimethyl sulfoxide molecule (point group symmetry $m$.), giving an irregular coordination geometry $[\mathrm{Ca}-\mathrm{O}$ bond length range $=2.345(2)-2.524(2) \AA]$. One-dimensional coordination complex chains extending parallel to $c$ are generated in which the triply $\mu_{2}-\mathrm{O}-$ bridged $\mathrm{Ca}^{2+}$ cations are separated by $3.6401(5) \AA$. In the crystal, weak intrachain $\mathrm{C}-\mathrm{H} \cdots \pi$ hydrogen bonds are present between the methyl $\mathrm{H}$ atoms of the dimethyl sulfoxide molecules as donors and the aromatic rings as acceptors $[\mathrm{C}-\mathrm{H} \cdots \mathrm{Cg}=3.790(4) \AA]$.

\section{Chemical context}

Compounds of benzoic acid with calcium are of special interest due to their wide-ranging applications, for example as a preservative in the food industry, in cosmetics and in medicine. In spite of that, the crystal structures of such compounds have been poorly investigated. Searches of the Cambridge Structural Database (CSD; Version 5.35, November 2013 + 2 updates; Groom \& Allen, 2014) for simple calcium benzoate complexes revealed only three results: $\left[\mathrm{Ca}(\text { benz })_{2}(\mathrm{dmf})\left(\mathrm{H}_{2} \mathrm{O}\right)\right]_{n}$ (Yano et al., 2001), $\left\{\left[\mathrm{Ca}(\text { benz })\left(\mathrm{H}_{2} \mathrm{O}\right)_{3}\right]^{+} \text {(benz) }{ }^{-}\right\}_{n}$ (Senkovska \& Thewalt, 2005) and $\left[\mathrm{Ca}(\text { benz })_{2}(\mathrm{Hbenz})\left(\mathrm{H}_{2} \mathrm{O}\right)\right]_{n}$ (Azizov et al., 2011) (where benz $=$ benzoate $)$.

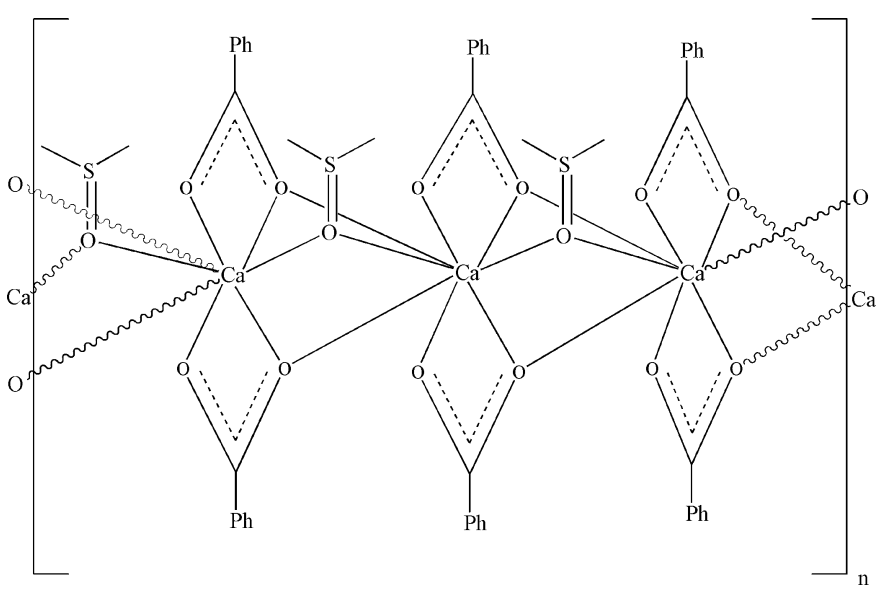

Here we report the synthesis of a new calcium benzoatedimethyl sulfoxide complex, $\left[\mathrm{Ca}(\text { benz })_{2}(\mathrm{dmso})\right]_{n}$, which was obtained as a by-product of an attempted synthesis of an $\mathrm{Mn}$ / 


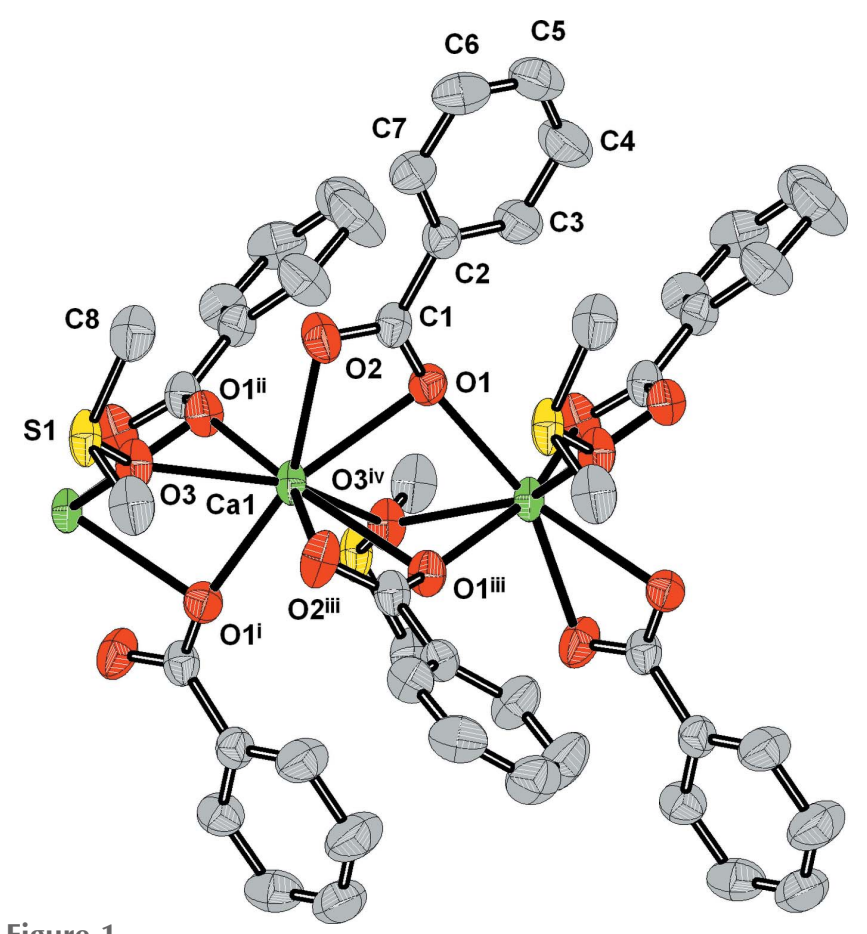

Figure 1

A fragment of the $\left[\mathrm{Ca}(\mathrm{benz})_{2}(\mathrm{dmso})\right]_{n}$ chain with the atom-labelling scheme. Displacement ellipsoids are drawn at the $50 \%$ probability level. $\mathrm{H}$ atoms have been omitted for clarity. For symmetry codes, see Table 1.

$\mathrm{Cu}$ heterometallic complex (in crystalline form available for $\mathrm{X}$-ray analysis) from the system: $\mathrm{Mn}-\mathrm{Cu}-(\mathrm{bhz}-\mathrm{sal})-\mathrm{CaO}-$ $\mathrm{KSCN}$-dmso (in open air), where manganese and copper were used as unactivated metal powders, bhz = benzohydrazide and sal = salicylaldehyde. The investigation of the system was carried out as a part of systematic research on the elaboration the 'direct synthesis' approach to both homo- and heterometallic coordination compounds (Babich et al., 1996; Buvaylo et al., 2005; Vassilyeva et al., 1997). It is worth noting that an alternative method of synthesis using a classical reaction between calcium oxide and benzoic acid in dmso, affords the same complex in good yield (up to $90 \%$ ), but does not give $\mathrm{X}$-ray quality crystals. The crystal structure of the title complex, $\left[\mathrm{Ca}(\text { benz })_{2}(\mathrm{dmso})\right]_{n}$, is reported herein.

\section{Structural commentary}

The asymmetric unit of $\left[\mathrm{Ca}(\text { benz })_{2}(\mathrm{dmso})\right]_{n}$ comprises one $\mathrm{Ca}^{2+}$ cation (site symmetry $m$..), one benzoate ligand and half

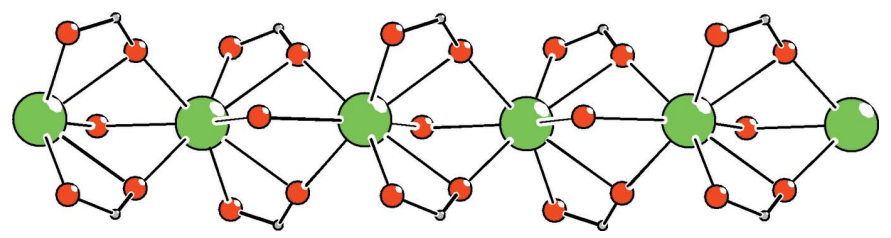

Figure 2

Bridging interactions observed in the title complex polymer which extends along the $c$ - axis direction. Phenyl rings and $\mathrm{H}$ atoms have been omitted for clarity.
Table 1

Selected bond lengths $(\AA)$.

\begin{tabular}{llll}
\hline $\mathrm{Ca} 1-\mathrm{O} 1^{\mathrm{i}}$ & $2.345(2)$ & $\mathrm{Ca} 1-\mathrm{O} 3$ & $2.494(3)$ \\
$\mathrm{Ca} 1-\mathrm{O} 1^{\mathrm{ii}}$ & $2.345(2)$ & $\mathrm{Ca} 1-\mathrm{O} 3^{\text {iv }}$ & $2.516(3)$ \\
$\mathrm{Ca} 1-\mathrm{O} 2^{\mathrm{iii}}$ & $2.481(2)$ & $\mathrm{Ca} 1-\mathrm{O} 1^{\mathrm{iii}}$ & $2.524(2)$ \\
$\mathrm{Ca} 1-\mathrm{O} 2$ & $2.481(2)$ & $\mathrm{Ca} 1-\mathrm{O} 1$ & $2.524(2)$ \\
\hline
\end{tabular}

Symmetry codes: (i) $-x+1,-y, z+\frac{1}{2}$; (ii) $x,-y, z+\frac{1}{2}$; (iii) $-x+1, y, z$; (iv) $-x+1,-y, z-\frac{1}{2}$.

Table 2

$\mathrm{C}-\mathrm{H} \cdots \pi$ interactions $\left(\AA{ }^{\circ}\right)$.

$\mathrm{Cg}$ is the centroid of the benzoate ring.

\begin{tabular}{lllll}
\hline$D-\mathrm{H} \cdots A$ & $D-\mathrm{H}$ & $\mathrm{H} \cdots A$ & $D \cdots A$ & $D-\mathrm{H} \cdots A$ \\
\hline $\mathrm{C} 8-\mathrm{H} 8 A \cdots C g$ & 0.96 & 2.84 & $3.790(4)$ & 169 \\
\hline
\end{tabular}

of a dmso molecule, the other half being generated by mirror symetry. The irregular $\mathrm{CaO}_{8}$ coordination polyhedron consists of six $\mathrm{O}$ atom donors from two $O, O^{\prime}$ chelating-bridging benzoate carboxyl groups with the same coordination modes, [2.1 $\left.1_{1} 1_{12}\right]$ in the Harris notation (Coxall et al., 2000), and two from $\mu_{2}$-bridging dmso molecules (Fig. 1). The coordination geometry deviates strongly from ideal, the $\mathrm{Ca}-\mathrm{O}$ bond lengths varying from 2.345 (2) to 2.524 (2) A (Table 1) and the $\mathrm{O}-\mathrm{Ca}-\mathrm{O}$ angles from $52.19(7)$ to $156.06(8)^{\circ}$. The bridging $\mathrm{Ca} 1-\mathrm{O} 1^{\mathrm{i}}$ and $\mathrm{Ca} 1-\mathrm{O} 1^{\mathrm{ii}}$ (carboxyl) bond lengths are considerably shorter than the chelate ones, as is usually observed in polymeric benzoates. For the title complex, the bond-valence index [BVS (Ca)] (Allmann, 1975) is 2.03.

\section{Supramolecular features}

The triple-O-bridged $\mathrm{CaO}_{8}$ polyhedra form one-dimensional coordination polymeric chains which extend parallel to the $c$ axis direction (Figs. 2-4). The $\mathrm{Ca} \cdot \mathrm{Ca}^{\mathrm{i}}$ and $\mathrm{Ca} 1 \cdots \mathrm{Ca} 1^{\text {iv }}$ separation in the chain is 3.6401 (5) $\AA$ [symmetry code (iv): $\left.-x+1,-y, z-\frac{1}{2}\right]$. To the best of our knowledge, this is the first $\mathrm{Ca}$ carboxylate polymer based on non-centrosymmetric bridges $\left(\mu-\eta^{2}: \eta^{1}\right)_{2}$. For bridging modes in coordination poly-

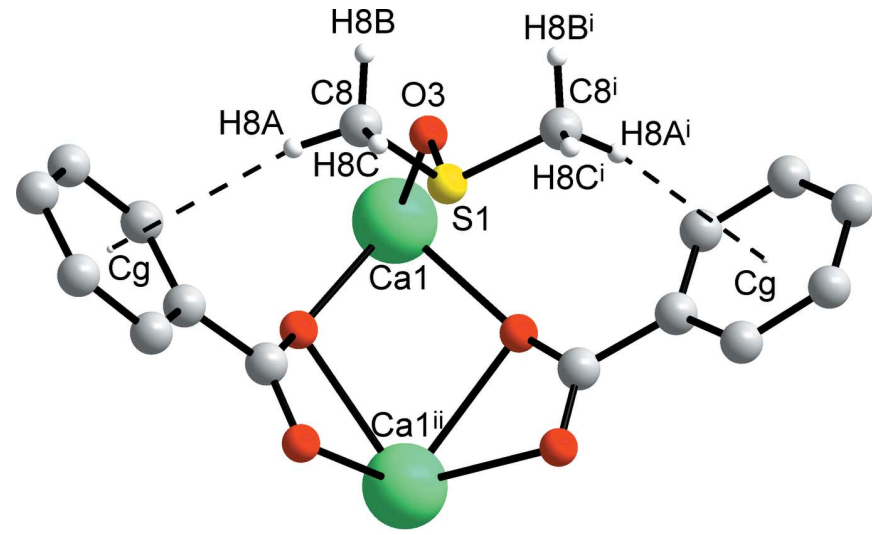

Figure 3

$\mathrm{C}-\mathrm{H} \cdots \pi$ hydrogen bonds involving a dmso donor as found in the title complex. For symmetry codes, see Table 1 ). 


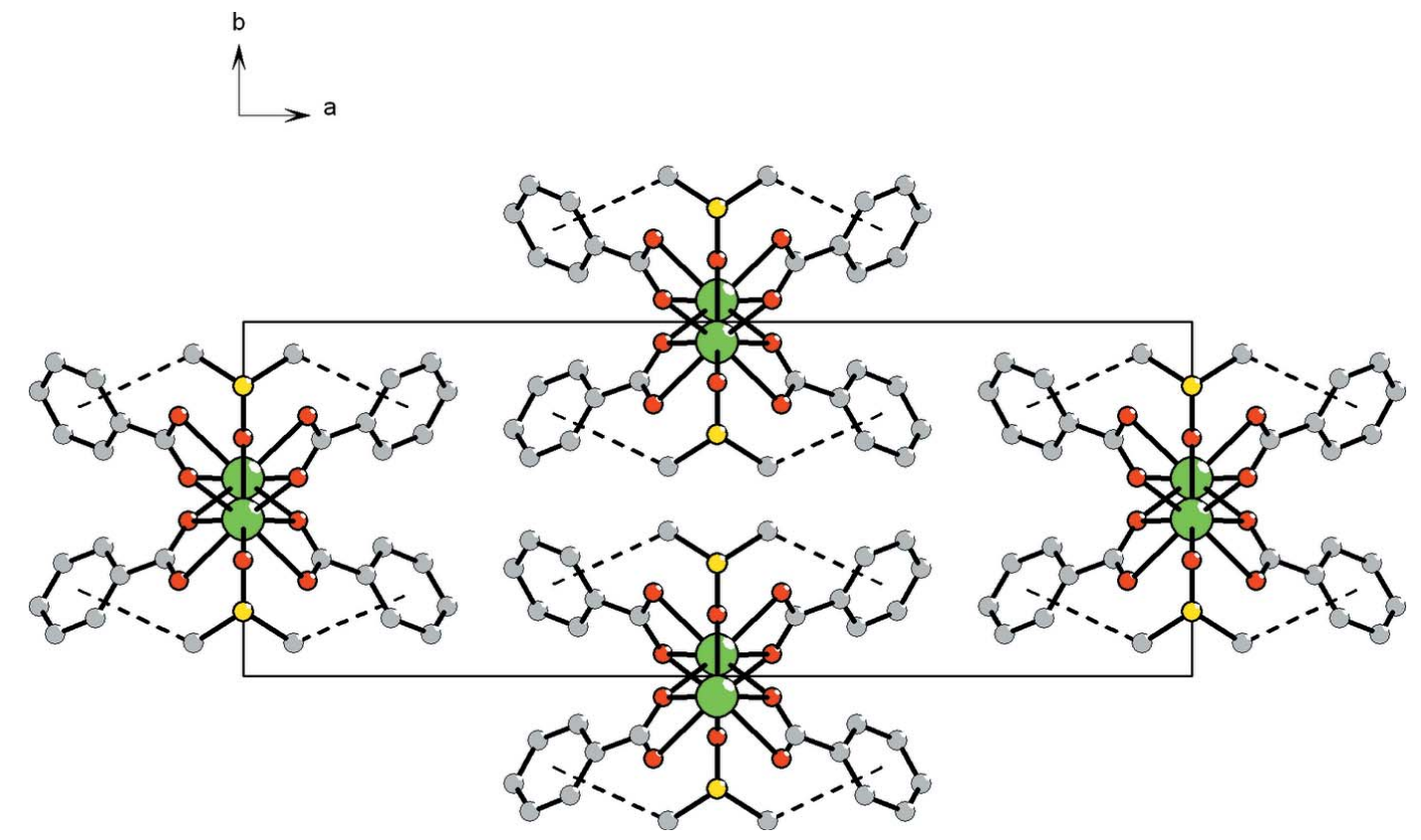

Figure 4

Packing of the molecular chains viewed down the chain direction (the crystallographic $c$ axis). $\mathrm{C}-\mathrm{H} \cdots \pi$ bonds are shown as dashed lines.

meric structures, reference should be made to Deacon et al. (2007) and Busskamp et al. (2007). The polymer chains in the title compound are additionally stabilized by weak $\mathrm{C}-\mathrm{H} \cdots \pi$ interactions between the methyl groups of the dmso molecule and the benzoate rings (centroid $C g$ ) (Table 2, Figs. 3 and 4).

\section{Synthesis and crystallization}

Calcium oxide $(0.056 \mathrm{~g}, 1 \mathrm{mmol})$ and benzoic acid $(0.244 \mathrm{~g}$, $2 \mathrm{mmol}$ ) were added to $20 \mathrm{ml}$ of dmso and stirred magnetically for $c a 5 \mathrm{~h}$ at $323 \mathrm{~K}$, after which the solution was filtered. The white precipitate which formed after one day was collected and dried in air; yield: $0.4 \mathrm{~g}$ (90\%). Elemental analysis for $\mathrm{C}_{16} \mathrm{H}_{16} \mathrm{CaO}_{5} \mathrm{~S}\left(M_{\mathrm{r}}=360.43\right)$. Calculated: $\mathrm{Ca}$, $11.12 \%$; found: $\mathrm{Ca}, 11.0 \%$. IR $\left(\mathrm{KBr}, \mathrm{cm}^{-1}\right): 1603(s), 1562(s)$, $1405(s), 1024(s), 721(s)$. Crystals suitable for X-ray analysis were obtained by slow evaporation at room temperature of a solution which was the product from the reaction between manganese powder $(0.05 \mathrm{~g}, 1 \mathrm{mmol})$, copper powder $(0.06 \mathrm{~g}$, $1 \mathrm{mmol})$, benzohydrazide $(0.409 \mathrm{~g}, 3 \mathrm{mmol})$, salicylaldehyde (0.314 ml, $3 \mathrm{mmol}), \mathrm{CaO}(0.168 \mathrm{~g}, 3 \mathrm{mmol}), \mathrm{KSCN}(0.291 \mathrm{~g}$, $3 \mathrm{mmol})$ and dmso $(20 \mathrm{ml})$. The reaction was carried out at $353 \mathrm{~K}$ with magnetic stirring for eight hours, after which undissolved products were filtered off.

\section{Refinement details}

Crystal data, data collection and structure refinement details are given in Table 3. Hydrogen atoms were placed in calculated positions $\left[\mathrm{C}-\mathrm{H}_{\text {aromatic }}=0.95 ; \mathrm{C}-\mathrm{H}_{\text {methyl }}=0.99 \AA\right]$ and were allowed to ride in the refinements, with $U_{\text {iso }}(\mathrm{H})=$ $1.2 U_{\text {eq }}$ (aromatic $\mathrm{C}$ ) or $1.5 U_{\text {eq }}$ (methyl $\mathrm{C}$ ). Although not of relevance in this crystal involving achiral molecules, the Flack absolute structure parameter (Flack, 1983) was determined as 0.04 (8) by classical fit to all intensities and 0.07 (3) from 557 selected quotients (Parsons et al., 2013).

Table 3

Experimental details.

\begin{tabular}{|c|c|}
\hline \multicolumn{2}{|l|}{ Crystal data } \\
\hline Chemical formula & {$\left[\mathrm{Ca}\left(\mathrm{C}_{7} \mathrm{H}_{5} \mathrm{O}_{2}\right)_{2}\left(\mathrm{C}_{2} \mathrm{H}_{6} \mathrm{OS}\right)\right]$} \\
\hline$M_{\mathrm{r}}$ & 360.43 \\
\hline Crystal system, space group & Orthorhombic, $C m c 2_{1}$ \\
\hline Temperature $(\mathrm{K})$ & 200 \\
\hline$a, b, c(\AA)$ & $25.531(2), 9.5351(8), 6.9330(4)$ \\
\hline$V\left(\AA^{3}\right)$ & $1687.7(2)$ \\
\hline$Z$ & 4 \\
\hline Radiation type & Мо $K \alpha$ \\
\hline$\mu\left(\mathrm{mm}^{-1}\right)$ & 0.52 \\
\hline Crystal size $(\mathrm{mm})$ & $0.22 \times 0.15 \times 0.11$ \\
\hline \multicolumn{2}{|l|}{ Data collection } \\
\hline Diffractometer & Rigaku Mercury CCD \\
\hline Absorption correction & Multi-scan (Blessing, 1995) \\
\hline$T_{\min }, T_{\max }$ & $0.798,0.951$ \\
\hline $\begin{array}{l}\text { No. of measured, independent and } \\
\text { observed }[I>2 \sigma(I)] \text { reflections }\end{array}$ & $3704,1897,1676$ \\
\hline$R_{\text {int }}$ & 0.025 \\
\hline$(\sin \theta / \lambda)_{\max }\left(\AA^{-1}\right)$ & 0.683 \\
\hline \multicolumn{2}{|l|}{ Refinement } \\
\hline$R\left[F^{2}>2 \sigma\left(F^{2}\right)\right], w R\left(F^{2}\right), S$ & $0.044,0.103,1.13$ \\
\hline No. of reflections & 1897 \\
\hline No. of parameters & 109 \\
\hline No. of restraints & 1 \\
\hline H-atom treatment & H-atom parameters constrained \\
\hline$\Delta \rho_{\max }, \Delta \rho_{\min }\left(\mathrm{e} \AA^{-3}\right)$ & $0.59,-0.39$ \\
\hline Absolute structure & $\begin{array}{l}\text { Flack } x \text { determined using } 557 \\
\text { quotients }\left[\left(I^{+}\right)-\left(I^{-}\right)\right] /\left[\left(I^{+}\right)+\left(I^{-}\right)\right] \\
\quad \text { (Parsons } \text { et al., 2013) }\end{array}$ \\
\hline Absolute structure parameter & $0.07(3)$ \\
\hline
\end{tabular}

Computer programs: CrystalClear (Rigaku, 1999), SIR92 (Altomare et al., 1993)., SHELXL97 (Sheldrick, 2008), DIAMOND (Brandenburg \& Putz, 2006) and WinGX (Farrugia, 2012). 


\section{Acknowledgements}

This work was partly supported by the State Fund for Fundamental Research of Ukraine (project 54.3/005).

\section{References}

Allmann, R. (1975). Monatsh. Chem. 106, 779-793.

Altomare, A., Cascarano, G., Giacovazzo, C. \& Guagliardi, A. (1993). J. Appl. Cryst. 26, 343-350.

Azizov, O., Kadirova, Z., Azizov, T., Tolipov, S. \& Ibragimov, B. (2011). Acta Cryst. E67, m597.

Babich, O. A., Kokozay, N. V. \& Pavlenko, V. A. (1996). Polyhedron, 15, 2727-2731.

Blessing, R. H. (1995). Acta Cryst. A51, 33-38.

Brandenburg, K. \& Putz, H. (2006). DIAMOND. Crystal Impact GbR, Bonn, Germany.

Busskamp, H., Deacon, G. B., Hilder, M., Junk, P. C., Kynast, U. H., Lee, W. \& Turner, D. R. (2007). CrystEngComm, 9, 394-411.
Buvaylo, E. A., Kokozay, V. N., Vassilyeva, O. Yu., Skelton, B. W., Jezierska, J., Brunel, L. C. \& Ozarowski, A. (2005). Chem. Commun. pp. 4976-4978.

Coxall, R. A., Harris, S. G., Henderson, D. K., Parsons, S., Tasker, P. A. \& Winpenny, R. E. P. (2000). J. Chem. Soc. Dalton Trans. pp. 2349-2356.

Deacon, G. B., Hein, S., Junk, P. C., Jüstel, T., Lee, W. \& Turner, D. R. (2007). CrystEngComm, 9, 1110-1123.

Farrugia, L. J. (2012). J. Appl. Cryst. 45, 849-854.

Flack, H. D. (1983). Acta Cryst. A39, 876-881.

Groom, C. R. \& Allen, F. H. (2014). Angew. Chem. Int. Ed. 53, 662671.

Parsons, S., Flack, H. D. \& Wagner, T. (2013). Acta Cryst. B69, 249259.

Rigaku (1999). CrystalClear. Rigaku Corporation, Tokyo, Japan.

Senkovska, I. \& Thewalt, U. (2005). Acta Cryst. C61, m448-m449.

Sheldrick, G. M. (2008). Acta Cryst. A64, 112-122.

Vassilyeva, O. Yu., Kokozay, V. N., Zhukova, N. I. \& Kovbasyuk, L. A. (1997). Polyhedron, 16, 263-266.

Yano, S., Numata, M., Kato, M., Motoo, S. \& Nishimura, T. (2001). Acta Cryst. E57, m488-m490. 


\section{supporting information}

Acta Cryst. (2015). E71, 906-909 [https://doi.org/10.1107/S2056989015012487]

\section{Crystal structure of catena-poly[calcium-di- $\mu_{3}$-benzoato- $\kappa^{6} O, O^{\prime}: O-\mu_{2}$-(dimethyl sulfoxide) $\left.-\kappa^{2} O: O\right]$}

\section{Anna S. Voronova, Svitlana R. Petrusenko and Evgeny Goreshnik}

Computing details

Data collection: CrystalClear (Rigaku, 1999); cell refinement: CrystalClear (Rigaku, 1999); data reduction: CrystalClear (Rigaku, 1999); program(s) used to solve structure: SIR92 (Altomare et al., 1993).; program(s) used to refine structure: SHELXL97 (Sheldrick, 2008); molecular graphics: DIAMOND (Brandenburg \& Putz, 2006); software used to prepare material for publication: $\operatorname{Win} G X$ (Farrugia, 2012).

catena-Poly[calcium-di- $\mu_{3}$-benzoato- $\kappa^{6} O, O^{\prime}: O-\mu_{2}$-(dimethyl sulfoxide)- $\kappa^{2} O: O$ ]

\section{Crystal data}

$\left[\mathrm{Ca}\left(\mathrm{C}_{7} \mathrm{H}_{5} \mathrm{O}_{2}\right)_{2}\left(\mathrm{C}_{2} \mathrm{H}_{6} \mathrm{OS}\right)\right]$

$M_{r}=360.43$

Orthorhombic, $C m c 2_{1}$

$a=25.531(2) \AA$

$b=9.5351(8) \AA$

$c=6.9330(4) \AA$

$V=1687.7(2) \AA^{3}$

$Z=4$

$F(000)=752$

Data collection

Rigaku Mercury CCD (2x2 bin mode) diffractometer

Graphite monochromator

Detector resolution: 14.7059 pixels $\mathrm{mm}^{-1}$

dtprofit.ref scans

Absorption correction: multi-scan (Blessing, 1995)

$T_{\min }=0.798, T_{\max }=0.951$

\section{Refinement}

Refinement on $F^{2}$

Least-squares matrix: full

$R\left[F^{2}>2 \sigma\left(F^{2}\right)\right]=0.044$

$w R\left(F^{2}\right)=0.103$

$S=1.13$

1897 reflections

109 parameters

1 restraint

Primary atom site location: structure-invariant direct methods
$D_{\mathrm{x}}=1.418 \mathrm{Mg} \mathrm{m}^{-3}$

Mo $K \alpha$ radiation, $\lambda=0.71069 \AA$

Cell parameters from 1872 reflections

$\theta=2.3-28.7^{\circ}$

$\mu=0.52 \mathrm{~mm}^{-1}$

$T=200 \mathrm{~K}$

Block, colorless

$0.22 \times 0.15 \times 0.11 \mathrm{~mm}$

3704 measured reflections

1897 independent reflections

1676 reflections with $I>2 \sigma(I)$

$R_{\text {int }}=0.025$

$\theta_{\max }=29.0^{\circ}, \theta_{\min }=2.3^{\circ}$

$h=-34 \rightarrow 26$

$k=-12 \rightarrow 6$

$l=-9 \rightarrow 9$

Secondary atom site location: difference Fourier map

Hydrogen site location: inferred from neighbouring sites

$\mathrm{H}$-atom parameters constrained

$w=1 /\left[\sigma^{2}\left(F_{\mathrm{o}}^{2}\right)+(0.040 P)^{2}+0.7932 P\right]$

where $P=\left(F_{\mathrm{o}}{ }^{2}+2 F_{\mathrm{c}}{ }^{2}\right) / 3$

$(\Delta / \sigma)_{\max }<0.001$

$\Delta \rho_{\max }=0.59 \mathrm{e} \AA^{-3}$

$\Delta \rho_{\min }=-0.39$ e $\AA^{-3}$ 
Absolute structure: Flack $x$ determined using 557 quotients $\left[\left(I^{+}\right)-(I)\right] /\left[\left(I^{+}\right)+\left(I^{-}\right)\right]$(Parsons et al., 2013)

Absolute structure parameter: 0.07 (3)

\section{Special details}

Geometry. All e.s.d.'s (except the e.s.d. in the dihedral angle between two l.s. planes) are estimated using the full covariance matrix. The cell e.s.d.'s are taken into account individually in the estimation of e.s.d.'s in distances, angles and torsion angles; correlations between e.s.d.'s in cell parameters are only used when they are defined by crystal symmetry. An approximate (isotropic) treatment of cell e.s.d.'s is used for estimating e.s.d.'s involving 1.s. planes.

Refinement. Refinement of $F^{2}$ against ALL reflections. The weighted $R$-factor $w R$ and goodness of fit $S$ are based on $F^{2}$, conventional $R$-factors $R$ are based on $F$, with $F$ set to zero for negative $F^{2}$. The threshold expression of $F^{2}>\sigma\left(F^{2}\right)$ is used only for calculating $R$-factors (gt) etc. and is not relevant to the choice of reflections for refinement. $R$-factors based on $F^{2}$ are statistically about twice as large as those based on $F$, and $R$ - factors based on ALL data will be even larger.

Fractional atomic coordinates and isotropic or equivalent isotropic displacement parameters $\left(\AA^{2}\right)$

\begin{tabular}{lllll}
\hline & $x$ & $y$ & $z$ & $U_{\text {iso }} * U_{\text {eq }}$ \\
\hline Ca1 & 0.5000 & $0.05825(7)$ & $0.36175(11)$ & $0.0325(2)$ \\
O1 & $0.44205(8)$ & $0.06089(19)$ & $0.0668(3)$ & $0.0388(5)$ \\
O2 & $0.43213(9)$ & $0.2334(2)$ & $0.2748(4)$ & $0.0509(6)$ \\
O3 & 0.5000 & $0.1724(3)$ & $0.6855(5)$ & $0.0414(7)$ \\
C1 & $0.41769(12)$ & $0.1682(3)$ & $0.1275(5)$ & $0.0375(7)$ \\
C2 & $0.37045(12)$ & $0.2146(3)$ & $0.0171(5)$ & $0.0388(7)$ \\
C3 & $0.35274(13)$ & $0.1367(4)$ & $-0.1381(7)$ & $0.0589(10)$ \\
H3 & 0.3695 & 0.0533 & -0.1704 & $0.071^{*}$ \\
C4 & $0.31036(16)$ & $0.1818(5)$ & $-0.2452(8)$ & $0.0756(13)$ \\
H4 & 0.2988 & 0.1289 & -0.3495 & $0.091^{*}$ \\
C5 & $0.28529(14)$ & $0.3044(4)$ & $-0.1984(7)$ & $0.0624(11)$ \\
H5 & 0.2567 & 0.3340 & -0.2707 & $0.075^{*}$ \\
C6 & $0.30222(15)$ & $0.3831(4)$ & $-0.0461(7)$ & $0.0584(11)$ \\
H6 & 0.2855 & 0.4669 & -0.0160 & $0.070^{*}$ \\
C7 & $0.34453(12)$ & $0.3375(3)$ & $0.0639(6)$ & $0.0464(8)$ \\
H7 & 0.3555 & 0.3900 & 0.1695 & $0.056^{*}$ \\
C8 & $0.44725(16)$ & $0.4090(3)$ & $0.6588(6)$ & $0.0560(10)$ \\
H8A & 0.4146 & 0.3721 & 0.7051 & $0.084^{*}$ \\
H8B & 0.4493 & 0.3964 & 0.5217 & $0.084^{*}$ \\
H8C & 0.4494 & 0.5071 & 0.6889 & $0.084^{*}$ \\
S1 & 0.5000 & $0.31863(10)$ & $0.77164(16)$ & $0.0444(3)$ \\
& & & &
\end{tabular}

Atomic displacement parameters $\left(\AA^{2}\right)$

\begin{tabular}{lllllll}
\hline & $U^{11}$ & $U^{22}$ & $U^{33}$ & $U^{12}$ & $U^{13}$ & $U^{23}$ \\
\hline Ca1 & $0.0504(4)$ & $0.0257(3)$ & $0.0214(4)$ & 0.000 & 0.000 & $0.0005(3)$ \\
O1 & $0.0472(11)$ & $0.0343(11)$ & $0.0349(13)$ & $0.0085(8)$ & $0.0000(10)$ & $-0.0033(9)$ \\
O2 & $0.0720(14)$ & $0.0427(12)$ & $0.0381(13)$ & $0.0142(11)$ & $-0.0139(13)$ & $-0.0064(11)$ \\
O3 & $0.069(2)$ & $0.0218(12)$ & $0.0333(17)$ & 0.000 & 0.000 & $-0.0020(12)$ \\
C1 & $0.0515(17)$ & $0.0302(14)$ & $0.0307(17)$ & $0.0017(13)$ & $0.0019(13)$ & $0.0022(12)$ \\
C2 & $0.0421(15)$ & $0.0389(16)$ & $0.0353(17)$ & $0.0002(13)$ & $0.0011(13)$ & $0.0042(14)$
\end{tabular}




\begin{tabular}{lllllll} 
C3 & $0.0556(18)$ & $0.060(2)$ & $0.061(2)$ & $0.0180(16)$ & $-0.014(2)$ & $-0.022(2)$ \\
C4 & $0.067(2)$ & $0.086(3)$ & $0.074(3)$ & $0.020(2)$ & $-0.031(2)$ & $-0.022(3)$ \\
C5 & $0.0483(19)$ & $0.069(2)$ & $0.070(3)$ & $0.0101(19)$ & $-0.0107(18)$ & $0.010(2)$ \\
C6 & $0.0478(19)$ & $0.045(2)$ & $0.082(3)$ & $0.0097(16)$ & $0.0054(19)$ & $0.003(2)$ \\
C7 & $0.0483(17)$ & $0.0390(17)$ & $0.052(2)$ & $0.0063(14)$ & $0.0025(16)$ & $-0.0028(16)$ \\
C8 & $0.083(3)$ & $0.0357(15)$ & $0.049(2)$ & $0.0096(17)$ & $0.005(2)$ & $-0.0010(17)$ \\
S1 & $0.0829(8)$ & $0.0257(5)$ & $0.0244(6)$ & 0.000 & 0.000 & $-0.0015(4)$ \\
\hline
\end{tabular}

Geometric parameters $\left(\AA,{ }^{\circ}\right)$

\begin{tabular}{|c|c|c|c|}
\hline $\mathrm{Ca} 1-\mathrm{O}^{\mathrm{i}}$ & $2.345(2)$ & $\mathrm{C} 1-\mathrm{C} 2$ & $1.496(4)$ \\
\hline $\mathrm{Ca} 1-\mathrm{O} 1^{\mathrm{ii}}$ & $2.345(2)$ & $\mathrm{C} 2-\mathrm{C} 3$ & $1.383(5)$ \\
\hline $\mathrm{Ca} 1-\mathrm{O} 2^{\mathrm{iii}}$ & $2.481(2)$ & $\mathrm{C} 2-\mathrm{C} 7$ & $1.384(4)$ \\
\hline $\mathrm{Ca} 1-\mathrm{O} 2$ & $2.481(2)$ & $\mathrm{C} 3-\mathrm{C} 4$ & $1.381(5)$ \\
\hline $\mathrm{Ca} 1-\mathrm{O} 3$ & $2.494(3)$ & $\mathrm{C} 3-\mathrm{H} 3$ & 0.9300 \\
\hline $\mathrm{Ca} 1-\mathrm{O}^{\text {iv }}$ & $2.516(3)$ & $\mathrm{C} 4-\mathrm{C} 5$ & $1.371(5)$ \\
\hline $\mathrm{Ca} 1-\mathrm{O} 1^{\mathrm{iii}}$ & $2.524(2)$ & $\mathrm{C} 4-\mathrm{H} 4$ & 0.9300 \\
\hline $\mathrm{Ca} 1-\mathrm{O} 1$ & $2.524(2)$ & $\mathrm{C} 5-\mathrm{C} 6$ & $1.365(6)$ \\
\hline $\mathrm{Ca} 1-\mathrm{C} 1^{\mathrm{iii}}$ & $2.855(3)$ & $\mathrm{C} 5-\mathrm{H} 5$ & 0.9300 \\
\hline $\mathrm{Ca} 1-\mathrm{C} 1$ & $2.855(3)$ & $\mathrm{C} 6-\mathrm{C} 7$ & $1.392(5)$ \\
\hline $\mathrm{Ca} 1-\mathrm{Ca} 1^{\mathrm{i}}$ & $3.6401(5)$ & C6- $\mathrm{H} 6$ & 0.9300 \\
\hline $\mathrm{Ca} 1-\mathrm{Ca} 1^{\mathrm{iv}}$ & $3.6401(5)$ & $\mathrm{C} 7-\mathrm{H} 7$ & 0.9300 \\
\hline $\mathrm{O} 1-\mathrm{C} 1$ & $1.269(3)$ & $\mathrm{C} 8-\mathrm{S} 1$ & $1.780(4)$ \\
\hline $\mathrm{O} 1-\mathrm{Ca}^{\mathrm{iv}}$ & $2.345(2)$ & $\mathrm{C} 8-\mathrm{H} 8 \mathrm{~A}$ & 0.9600 \\
\hline $\mathrm{O} 2-\mathrm{C} 1$ & $1.251(4)$ & $\mathrm{C} 8-\mathrm{H} 8 \mathrm{~B}$ & 0.9600 \\
\hline $\mathrm{O} 3-\mathrm{S} 1$ & $1.517(3)$ & $\mathrm{C} 8-\mathrm{H} 8 \mathrm{C}$ & 0.9600 \\
\hline $\mathrm{O} 3-\mathrm{Ca} 1^{\mathrm{i}}$ & $2.516(3)$ & $\mathrm{S} 1-\mathrm{C} 8^{\mathrm{iii}}$ & $1.780(4)$ \\
\hline $\mathrm{O} 1^{\mathrm{i}}-\mathrm{Ca} 1-\mathrm{O} 1^{\mathrm{ii}}$ & $78.22(11)$ & $\mathrm{C} 11^{\mathrm{ii}}-\mathrm{Ca} 1-\mathrm{Ca} 1^{\mathrm{i}}$ & $130.82(7)$ \\
\hline $\mathrm{O} 1^{\mathrm{i}}-\mathrm{Ca} 1-\mathrm{O} 2^{\mathrm{iii}}$ & $91.88(8)$ & $\mathrm{C} 1-\mathrm{Ca} 1-\mathrm{Ca} 1^{\mathrm{i}}$ & $130.82(7)$ \\
\hline 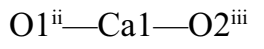 & $156.06(8)$ & $\mathrm{O} 1^{\mathrm{i}}-\mathrm{Ca} 1-\mathrm{Ca} 1^{\mathrm{iv}}$ & $115.44(6)$ \\
\hline $\mathrm{O} 1-\mathrm{Ca} 1-\mathrm{O} 2$ & $156.06(8)$ & $\mathrm{O} 1^{\mathrm{ii}}-\mathrm{Ca} 1-\mathrm{Ca} 1^{\mathrm{iv}}$ & $115.44(6)$ \\
\hline $\mathrm{O} 1{ }^{\mathrm{ii}}-\mathrm{Ca} 1-\mathrm{O} 2$ & $91.88(8)$ & $\mathrm{O} 2^{\mathrm{iii}}-\mathrm{Ca} 1-\mathrm{Ca} 1^{\mathrm{iv}}$ & $88.51(6)$ \\
\hline $\mathrm{O} 22^{\mathrm{iii}}-\mathrm{Ca} 1-\mathrm{O} 2$ & $88.60(12)$ & $\mathrm{O} 2-\mathrm{Ca} 1-\mathrm{Ca} 1^{\mathrm{iv}}$ & $88.51(6)$ \\
\hline $\mathrm{O} 1-\mathrm{Ca} 1-\mathrm{O} 3$ & $70.48(7)$ & $\mathrm{O} 3-\mathrm{Ca} 1-\mathrm{Ca} 1^{\mathrm{iv}}$ & $171.90(7)$ \\
\hline $\mathrm{O} 1{ }^{\mathrm{ii}}-\mathrm{Ca} 1-\mathrm{O} 3$ & $70.48(7)$ & $\mathrm{O}^{\mathrm{iv}}-\mathrm{Ca} 1-\mathrm{Ca} 1^{\mathrm{iv}}$ & $43.17(8)$ \\
\hline $\mathrm{O} 22^{\mathrm{iii}}-\mathrm{Ca} 1-\mathrm{O} 3$ & $85.70(8)$ & $\mathrm{O} 1^{\mathrm{iii}}-\mathrm{Ca} 1-\mathrm{Ca} 1^{\mathrm{iv}}$ & $39.79(5)$ \\
\hline $\mathrm{O} 2-\mathrm{Ca} 1-\mathrm{O} 3$ & $85.70(8)$ & $\mathrm{O} 1-\mathrm{Ca} 1-\mathrm{Ca} 1^{\mathrm{iv}}$ & $39.79(5)$ \\
\hline $\mathrm{O} 1^{\mathrm{i}}-\mathrm{Ca} 1-\mathrm{O}^{\mathrm{iv}}$ & $82.59(8)$ & $\mathrm{C} 1^{\mathrm{iii}}-\mathrm{Ca} 1-\mathrm{Ca} 1^{\mathrm{iv}}$ & $64.56(7)$ \\
\hline $\mathrm{O} 1^{\mathrm{ii}}-\mathrm{Ca} 1-\mathrm{O}^{\mathrm{iv}}$ & $82.59(8)$ & $\mathrm{C} 1-\mathrm{Ca} 1-\mathrm{Ca} 1^{\mathrm{iv}}$ & $64.56(7)$ \\
\hline $\mathrm{O} 2^{\mathrm{iii}}-\mathrm{Ca} 1-\mathrm{O}^{\mathrm{iv}}$ & $118.06(7)$ & $\mathrm{Ca} 1^{\mathrm{i}}-\mathrm{Ca} 1-\mathrm{Ca} 1^{\mathrm{iv}}$ & 144.47 (4) \\
\hline $\mathrm{O} 2-\mathrm{Ca} 1-\mathrm{O}^{\mathrm{iv}}$ & $118.06(7)$ & $\mathrm{C} 1-\mathrm{O} 1-\mathrm{Ca}^{\text {iv }}$ & $154.2(2)$ \\
\hline $\mathrm{O} 3-\mathrm{Ca} 1-\mathrm{O}^{\mathrm{iv}}$ & $144.93(11)$ & $\mathrm{C} 1-\mathrm{O} 1-\mathrm{Ca} 1$ & 91.54 (19) \\
\hline 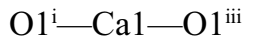 & $97.25(7)$ & $\mathrm{Ca}^{\mathrm{iv}}-\mathrm{O} 1-\mathrm{Ca} 1$ & $96.69(7)$ \\
\hline $\mathrm{O} 1^{\mathrm{ii}}-\mathrm{Ca} 1-\mathrm{O} 1^{\mathrm{iii}}$ & $149.97(5)$ & $\mathrm{C} 1-\mathrm{O} 2-\mathrm{Ca} 1$ & $94.01(18)$ \\
\hline $\mathrm{O} 2^{\mathrm{iii}}-\mathrm{Ca} 1-\mathrm{O} 1^{\mathrm{iii}}$ & $52.19(7)$ & $\mathrm{S} 1-\mathrm{O} 3-\mathrm{Ca} 1$ & $139.05(18)$ \\
\hline $\mathrm{O} 2-\mathrm{Ca} 1-\mathrm{O} 1^{\mathrm{iii}}$ & $101.88(8)$ & $\mathrm{S} 1-\mathrm{O} 3-\mathrm{Ca} 1^{\mathrm{i}}$ & $127.75(19)$ \\
\hline $\mathrm{O} 3-\mathrm{Ca} 1-\mathrm{O} 1^{\mathrm{iii}}$ & $136.43(6)$ & $\mathrm{Ca} 1-\mathrm{O} 3-\mathrm{Ca}^{\mathrm{i}}$ & $93.19(9)$ \\
\hline
\end{tabular}




\begin{tabular}{|c|c|c|c|}
\hline $\mathrm{O}^{\mathrm{iv}}-\mathrm{Ca} 1-\mathrm{O} 1^{\mathrm{iii}}$ & $67.37(7)$ & $\mathrm{O} 2-\mathrm{C} 1-\mathrm{O} 1$ & $121.8(3)$ \\
\hline $\mathrm{O} 1{ }^{\mathrm{i}}-\mathrm{Ca} 1-\mathrm{O} 1$ & $149.97(5)$ & $\mathrm{O} 2-\mathrm{C} 1-\mathrm{C} 2$ & $120.6(3)$ \\
\hline 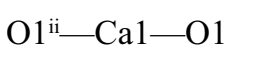 & $97.25(7)$ & $\mathrm{O} 1-\mathrm{C} 1-\mathrm{C} 2$ & $117.6(3)$ \\
\hline $\mathrm{O} 2^{\mathrm{iii}}-\mathrm{Ca} 1-\mathrm{O} 1$ & $101.88(8)$ & $\mathrm{O} 2-\mathrm{C} 1-\mathrm{Ca} 1$ & 60.08 (16) \\
\hline $\mathrm{O} 2-\mathrm{Ca} 1-\mathrm{O} 1$ & $52.19(7)$ & $\mathrm{O} 1-\mathrm{C} 1-\mathrm{Ca} 1$ & $62.08(16)$ \\
\hline $\mathrm{O} 3-\mathrm{Ca} 1-\mathrm{O} 1$ & $136.43(6)$ & $\mathrm{C} 2-\mathrm{C} 1-\mathrm{Ca} 1$ & $173.4(2)$ \\
\hline $\mathrm{O}^{\mathrm{iv}}-\mathrm{Ca} 1-\mathrm{O} 1$ & $67.37(7)$ & $\mathrm{C} 3-\mathrm{C} 2-\mathrm{C} 7$ & $118.8(3)$ \\
\hline $\mathrm{O} 1^{\mathrm{iii}}-\mathrm{Ca} 1-\mathrm{O} 1$ & $71.78(10)$ & $\mathrm{C} 3-\mathrm{C} 2-\mathrm{C} 1$ & $120.2(3)$ \\
\hline $\mathrm{O} 1^{\mathrm{i}}-\mathrm{Ca} 1-\mathrm{C} 1^{\mathrm{iii}}$ & $93.35(8)$ & $\mathrm{C} 7-\mathrm{C} 2-\mathrm{C} 1$ & $121.1(3)$ \\
\hline $\mathrm{O} 1^{\mathrm{ii}}-\mathrm{Ca} 1-\mathrm{C} 1^{\mathrm{iii}}$ & $170.72(8)$ & $\mathrm{C} 4-\mathrm{C} 3-\mathrm{C} 2$ & $120.4(3)$ \\
\hline 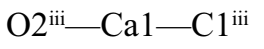 & $25.91(8)$ & $\mathrm{C} 4-\mathrm{C} 3-\mathrm{H} 3$ & 119.8 \\
\hline $\mathrm{O} 2-\mathrm{Ca} 1-\mathrm{C} 1^{\mathrm{iii}}$ & $97.39(9)$ & $\mathrm{C} 2-\mathrm{C} 3-\mathrm{H} 3$ & 119.8 \\
\hline $\mathrm{O} 3-\mathrm{Ca} 1-\mathrm{C} 1^{\mathrm{iii}}$ & $110.58(8)$ & $\mathrm{C} 5-\mathrm{C} 4-\mathrm{C} 3$ & $120.3(4)$ \\
\hline $\mathrm{O}^{3 \mathrm{iv}}-\mathrm{Ca} 1-\mathrm{Cl}^{\mathrm{iii}}$ & 92.57 (8) & $\mathrm{C} 5-\mathrm{C} 4-\mathrm{H} 4$ & 119.9 \\
\hline 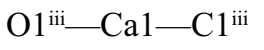 & $26.38(7)$ & $\mathrm{C} 3-\mathrm{C} 4-\mathrm{H} 4$ & 119.9 \\
\hline $\mathrm{O} 1-\mathrm{Ca} 1-\mathrm{C} 1^{\mathrm{iii}}$ & $88.11(8)$ & $\mathrm{C} 6-\mathrm{C} 5-\mathrm{C} 4$ & $120.2(4)$ \\
\hline $\mathrm{O} 1-\mathrm{Ca} 1-\mathrm{C} 1$ & $170.72(8)$ & $\mathrm{C} 6-\mathrm{C} 5-\mathrm{H} 5$ & 119.9 \\
\hline $\mathrm{O} 1^{\mathrm{ii}}-\mathrm{Ca} 1-\mathrm{C} 1$ & $93.35(8)$ & $\mathrm{C} 4-\mathrm{C} 5-\mathrm{H} 5$ & 119.9 \\
\hline $\mathrm{O} 2^{\mathrm{iii}}-\mathrm{Ca} 1-\mathrm{C} 1$ & $97.39(9)$ & $\mathrm{C} 5-\mathrm{C} 6-\mathrm{C} 7$ & $119.9(3)$ \\
\hline $\mathrm{O} 2-\mathrm{Ca} 1-\mathrm{C} 1$ & $25.91(8)$ & $\mathrm{C} 5-\mathrm{C} 6-\mathrm{H} 6$ & 120.1 \\
\hline $\mathrm{O} 3-\mathrm{Ca} 1-\mathrm{C} 1$ & $110.58(8)$ & $\mathrm{C} 7-\mathrm{C} 6-\mathrm{H} 6$ & 120.1 \\
\hline $\mathrm{O}^{\mathrm{iv}}-\mathrm{Ca} 1-\mathrm{C} 1$ & $92.57(8)$ & $\mathrm{C} 2-\mathrm{C} 7-\mathrm{C} 6$ & $120.4(3)$ \\
\hline $\mathrm{O} 1^{\mathrm{iii}}-\mathrm{Ca} 1-\mathrm{C} 1$ & $88.11(8)$ & $\mathrm{C} 2-\mathrm{C} 7-\mathrm{H} 7$ & 119.8 \\
\hline $\mathrm{O} 1-\mathrm{Ca} 1-\mathrm{C} 1$ & $26.38(7)$ & $\mathrm{C} 6-\mathrm{C} 7-\mathrm{H} 7$ & 119.8 \\
\hline $\mathrm{C}{ }^{\mathrm{iii}}-\mathrm{Ca} 1-\mathrm{C} 1$ & 94.77 (13) & $\mathrm{S} 1-\mathrm{C} 8-\mathrm{H} 8 \mathrm{~A}$ & 109.5 \\
\hline 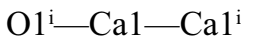 & $43.52(5)$ & $\mathrm{S} 1-\mathrm{C} 8-\mathrm{H} 8 \mathrm{~B}$ & 109.5 \\
\hline $\mathrm{O} 1^{\mathrm{ii}}-\mathrm{Ca} 1-\mathrm{Ca} 1^{\mathrm{i}}$ & $43.52(5)$ & $\mathrm{H} 8 \mathrm{~A}-\mathrm{C} 8-\mathrm{H} 8 \mathrm{~B}$ & 109.5 \\
\hline $\mathrm{O} 2^{\mathrm{iii}}-\mathrm{Ca} 1-\mathrm{Ca} 1^{\mathrm{i}}$ & $115.90(7)$ & $\mathrm{S} 1-\mathrm{C} 8-\mathrm{H} 8 \mathrm{C}$ & 109.5 \\
\hline $\mathrm{O} 2-\mathrm{Ca} 1-\mathrm{Ca} 1^{\mathrm{i}}$ & $115.90(7)$ & $\mathrm{H} 8 \mathrm{~A}-\mathrm{C} 8-\mathrm{H} 8 \mathrm{C}$ & 109.5 \\
\hline $\mathrm{O} 3-\mathrm{Ca} 1-\mathrm{Ca} 1^{\mathrm{i}}$ & $43.63(7)$ & $\mathrm{H} 8 \mathrm{~B}-\mathrm{C} 8-\mathrm{H} 8 \mathrm{C}$ & 109.5 \\
\hline $\mathrm{O}^{3 \mathrm{iv}}-\mathrm{Ca} 1-\mathrm{Ca} 1^{\mathrm{i}}$ & $101.29(8)$ & $\mathrm{O} 3-\mathrm{S} 1-\mathrm{C} 8^{\mathrm{iii}}$ & $105.78(14)$ \\
\hline $\mathrm{O} 1^{\mathrm{iii}}-\mathrm{Ca} 1-\mathrm{Ca} 1^{\mathrm{i}}$ & $140.76(5)$ & $\mathrm{O} 3-\mathrm{S} 1-\mathrm{C} 8$ & $105.78(14)$ \\
\hline $\mathrm{O} 1-\mathrm{Ca} 1-\mathrm{Ca} 1^{\mathrm{i}}$ & $140.76(5)$ & 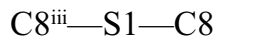 & $98.4(3)$ \\
\hline
\end{tabular}

Symmetry codes: (i) $-x+1,-y, z+1 / 2$; (ii) $x,-y, z+1 / 2$; (iii) $-x+1, y, z$; (iv) $-x+1,-y, z-1 / 2$.

Hydrogen-bond geometry $\left(\AA,{ }^{\circ}\right)$

$\mathrm{Cg}$ is the centroid of the benzoate ring.

\begin{tabular}{lllll}
\hline$D-\mathrm{H} \cdots A$ & $D-\mathrm{H}$ & $\mathrm{H} \cdots A$ & $D \cdots A$ & $D-\mathrm{H} \cdots A$ \\
\hline $\mathrm{C} 8-\mathrm{H} 8 A \cdots C g$ & 0.96 & 2.84 & $3.790(4)$ & 169 \\
\hline
\end{tabular}

